\title{
Journal of Real-Time Image Processing: third issue of volume 17
}

\author{
Nasser Kehtarnavaz ${ }^{1} \cdot$ Matthias F. Carlsohn ${ }^{2}$
}

Published online: 26 May 2020

(c) Springer-Verlag GmbH Germany, part of Springer Nature 2020

Within a short amount of time, the Covid-19 pandemic has changed the normal ways of living and work all over the world. The world is facing very difficult challenges generated by this pandemic. We wish to thank all frontline people, in particular healthcare professionals, for their sacrifices to fight this disease. To help the scientific community in this unprecedented time, Springer has opened up access to all published Springer content. We are grateful to Springer for their thoughtfulness in supporting the scientific activities of researchers, scholars, and students.

The editorial board of JRTIP was scheduled to meet during the SPIE Conference on Real-Time Image Processing and Deep Learning in Anaheim, CA, in April 2020 but due to the Covid-19 crisis, the meeting was cancelled. The editorial board is planning to have a virtual meeting online in the near future.

To give some idea of the journal performance in 2019, the journal received $550+$ manuscripts and published 92 papers. Also, the number of downloads of all the papers published in the journal reached over 83,000. The last impact factor of the journal (2018) stands at 2.588 and its five year impact factor stands at 2.378. We encourage authors to participate in the Springer conducted author surveys. More information on these surveys can be found at https://www.springernature. com/gp/authors/campaigns/journal-author-survey-results.

As mentioned in our previous editorials, in 2020, JRTIP has started a new publishing structure by having 6 issues per year with 15 or more articles per issue. So far in 2020, two issues, one special issue on "Real-Time Image Watermarking and Forensics in Cloud Computing" consisting of 16 articles, and one regular issue consisting of 14 articles, have been published. This third issue is a regular issue consisting of 25 articles, 15 regular articles followed by a special issue section. The special issue section includes 10 special issue articles that did not appear in the

\footnotetext{
Nasser Kehtarnavaz

kehtar@utdallas.edu

Matthias F. Carlsohn

Matthias.Carlsohn@t-online.de

University of Texas at Dallas, Richardson, TX, USA

2 Engineering and Consultancy for Computer Vision and Image Communication, Bremen, Germany
}

following three past special issues for various reasons: "Deep Learning for Real-Time Information Hiding and Forensics", "Real-Time Image and Video Processing in Mobile Embedded Systems", and "Real-Time Radar, Lidar, Video Wireless Imaging for Smart Vehicles and Intelligent Transportation Systems".

To give authors, reviewers, and editors time to adjust to the current pandemic situation, we have already extended all the deadlines associated with our active special issues by 90 days. The updated calls for papers for these special issues appear at the journal website and also in the backmatter of this issue.

We wish to remind authors that this journal is a member of the Committee on Publication Ethics (COPE) and urge authors to follow the ethical responsibilities listed at https://www.sprin ger.com/journal/11554/submission-guidelines\#Instructions $\% 20$ for\%20Authors_Ethical\%20Responsibilities\%20of\%20Authors.

In particular, it is important to emphasize that authors are strongly advised to ensure the author list is final and the order is correct at the submission time. This is to let authors know that we will not permit adding and/or deleting authors during the review process. If authors are added or deleted, we will pause the review process and refer the matter to Springer for examination. Strict rules are applied for any exceptions. Also, under no circumstances, no change to authorship is permitted after acceptance of manuscripts. A change in the order of authorship will be considered only if all the authors of a manuscript sign the authorship change form and state detailed reasons for changing the authorship order.

We would like to mention that the Associate Editors Antonio Plaza and Barak Fishbain are stepping down in their role as AEs due to the other obligations they have. We thank them for all their valuable contributions in conducting reviews of manuscripts and wish them well.

Finally, we express our thanks to our readers, authors, reviewers, editors, and Springer publishing team for their contributions and wish them health and safety in this challenging time. Let us hope that the Covid-19 pandemic goes away soon.

Publisher's Note Springer Nature remains neutral with regard to jurisdictional claims in published maps and institutional affiliations. 